\section{Artérias da base do encéfalo de cães (Canis familiaris, Linnaeus, 1758). II. Formação e comportamento do circuito arterial do encéfalo*}

\author{
Arteries of the basis of the encephalon in dogs (Canis familiaris, \\ Linnaeus, 1758). II. Formation and behaviour of the \\ encephalon arterial circuit
}

Maria Aparecida de AICÂATARA'; Irvênia Luiza de Santis PRADA
CORRESPONDENCE TO

Irvênia Luiza de Santis Prada

Departamento de Cirurgia

Faculdade de Medicina Veterinária e

Zootecnia da USP

Av. Prof. Dr. Orlando Marques de

Paiva, 87 - Cidade Universitária

"Armando de Salles Oliverra"

05508-900 - São Paulo - SP - Brasil

\title{
RESUMO
}

Foram estudadas, mediante dissecação, 30 peças de cães SRD (sem raça definida), com o objetivo de melhor conhecer particularidades do comportamento das artérias da base do encéfalo. Nestas preparações observou-se que a particular disposição dos ramos terminais das artérias carótidas internas e basilar determina a formação de um CIRCUITO ARTERIAL, que contorna a hipófise e o quiasma óptico. Esta formação, a partir da divisão da artéria carótida interna, de ambos os lados, em seus ramos terminais rostral e caudal, apresenta-se, de modo invariável, em pequeno arco ou ferradura de concavidade caudal; caudalmente, de forma variada, constitui figura piriforme (56,6\%) ou poligonal $(43,3 \%)$ representada rostralmente, pelas artérias cerebrais rostrais esquerda e direita; lateralmente, pelos ramos terminais rostral e caudal das artérias carótidas internas esquerda e direita; e caudolateralmente, pelos ramos terminais (à esquerda e à direita) da artéria basilar.

UNITERMOS: Anatomia; Cães; Cérebro; Artérias.

\section{INTRODUCÃO}

$\mathrm{D}$ urante estudo sistemático das artérias da base do encélalo de cães que efetuamos anteriormente (Alcântara; Prada, 1996)**, surpreendemo-nos com a particular disposição dos vasos que contornam a hipófise e o quiasma óptico, constituindo fïgura que não correspondia nem a círeulo -- "círculo arterial de Willis" -- como descrevem alguns autores, a exemplo de Nickel et al." (1975), nem tampouco a um polígono -- "polígono arterial de Willis" -- como referem outros, a exemplo de Baptista (1922). Devido à ausência de qualquer citação a respeito, na literatura consultada, compatível com a formação por nós encontrada, decidimos relatar essa particular disposição encontrada em cães e, assim, oportunamente, reavaliar conceitos até agora adotados para denominá-la.

\section{MATERIAL E METOOOO}

Esta pesquisa foi realizada junto às disciplinas de Ana-



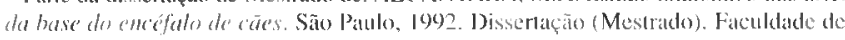
Medicina Velerinária e Zootecnia, Universidade de São Paulo.

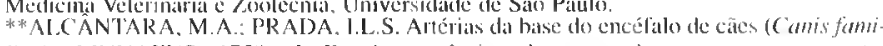
fiaris, I.INNAEUS, 1758). I. Estudo anatomico de suits origens e comporlamento Braziliam fournal Veterinany Research Science, v.33, n.2. 1996.

**** - Du Pont do Brasil S.A. - Indústrials Quimicas.

***** - Suvinil Corante - Gilasurit do Bratsil S.A
}

tomia dos Animais Domésticos do Departamento da Faculdade de Medicina Veterinária e Zootecnia da Universidade de São Paulo, com 30 cães SRD (sem raça definida), adultos, que foram previamente utilizados junto à disciplina de Técnica Cirúrgica ou que vieram a óbito no Hospital Veterinário desta Faculdade. Nestes cães canulamos a artéria carótida comum à esquerda ou à direita e, com auxílio de seringa de $50 \mathrm{ml}$, perfundimos o sistema vascular com água à temperatura de $23^{\circ} \mathrm{C}$ e. logo após, solução de Neoprene látex 45()*** corado em vermelho com pigmento especifico kv*k. $^{*}$. A fixação foi realizada mediante injeção de formol a $15 \%$, pela veia jugular externa de um dos lados.

Os termos utilizados para a designação das attérias estudadas, de modo geral, estão de acordo com a NOMINA ANATÔMICA VETERINÁRIA' (1983); diferentemente desta fonte usamos as denominações "Circuito Arterial do Encéfalo", "Ramo Rostral da Artéria Carólida Interna" e "Ramo Caudal da Artéria Carótida Interna", em substituição às expressões "Círculo Arterial Cerebral", "Artéria Cerebral Rostral" e "Artéria Comunicante Caudal", por julgarmos que assim defïnem com maior clareza as disposições encontradas.

Finalmente, para o estudo estatístico, aplicamos o cálculo de porcentagem e o teste de duas proporções com distribuição normal $(\alpha=5 \%)$. 


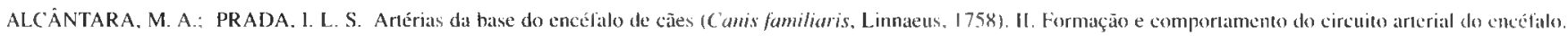

Braz. J. vet. Res. anim. Sci.. São Paulo, v.33. n.2. p.72-76. 1996.

\section{RESULTADOS}

\section{CIRCUITO ARTERIAL, DO ENCÉFALO}

(FICS. 1, 2, 3, 4)

Nas 30 peças dissecadas observamos, na base do encéfálo, que a particular disposição dos ramos terminais das artérias carótidas internas (direita e esquerda) e basilar, determina formação que contorna o quiasma óptico e a hipólïse. Essa formação, a partir da divisão da artéria carótida interna, de ambos os lados, em seus ramos terminais rostral e caudal, apresenta-se de modo invariável, rostralmente, em pequeno arco ou em ferradura de concavidade caudal e, caudalmente de forma variada. Esssa disposição em lerradura (Fig. I), que contorna o quiasma óptico, constituída à custa do ramo rostral da artéria carótida interna, de cada lado, continua-se rostralmente com a correspondente artéria cerebral roslral. Em 17 das peças $(56,6 \% \pm 9,1$ Fig. 1) caudalmente constitui-se figura piriforme cuja base relaciona-se aos pedúnculos cerebrais e. em outros 13 casos $(43,3 \% \pm 9,0$ Fig. 2), figura poligonal, 1omando-se o

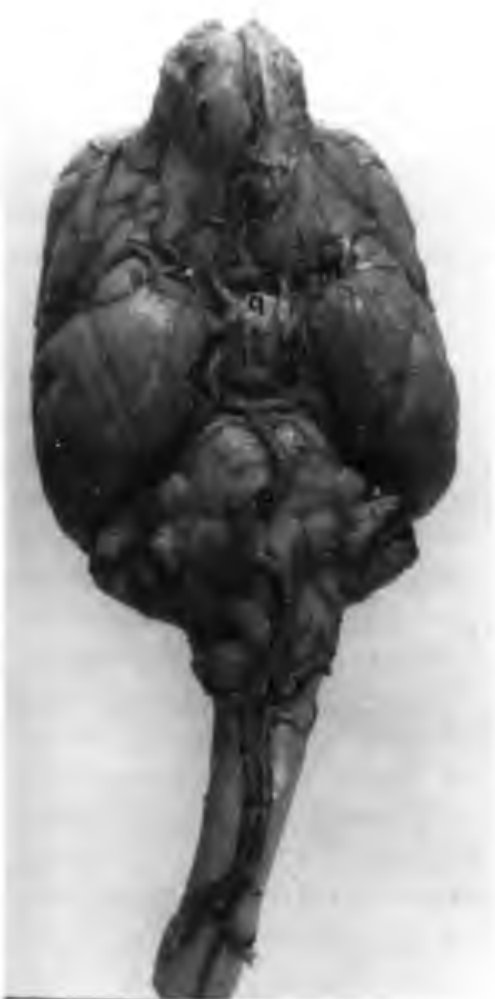
auxílio de linha imaginária colocada em correspondência à bifurcação da ałtéria carótida interna, de ambos os lados.

No grupo das 17 peças cuja fïgura é piriforme, destacamos 10 preparações $(33,3 \% \pm$ 8.6 Fig. 3) nas quais verificamos, na aludida figura, a ocorrência de ângulos pouco pronunciados. Esses ângulos, embora pouco pronunciados. es1ão presentes: de ambos os lados, em 2 ca$\operatorname{sos}(6,6 \% \pm 4.5$ Fig. 3), sendo que, em um deles encontramo-los em correspondência à origem da artéria cerebral caudal e da ar-

\section{Figura 1}

Encéfalo de cão sem raça definida -- vista ventral. Nesta preparação observa-se que a particular disposição dos ramos rostral e caudal (D) da artéria carótida interna, bem como dos ramos terminais ( $t$ ) da artéria basilar, determinam formação -- CIRCUITO ARTERIAL -- que contorna o quiasma óptico $(\mathrm{q})$ e a hipófise $(\mathrm{H})$ e se mostra, rostralmente, sempre em arco e, caudalmente, constituindo figura piriforme $(56,6 \%)$. Artéria Basilar (B). Artéria Cerebral Rostral (R). Artéria Cerebral caudal (D). Artéria cerebelar caudal $(\mathrm{g})$. Artéria espinhal ventral (e). Losango arterial (d). Artéria vertebral ( $v$ ).



Figura 2

Encéfalo de cão sem raça definida - Aumento de aproximadamente, $0.5 \mathrm{vez}$. O CIRCUITO ARTERIAL achase representado, caudalmente, por figura poligonal $(43,3 \%)$ traçada com auxilio de linha imaginária, colocada em correspondência à bifurcação da artéria carótida interna, de ambos os lados.

Identificamos, ainda, artéria basilar (B), seu ramo terminal $(t)$ unindo-se ao ramo caudal (D) da artéria carótida interna no limite referendado pela origem da artéria cerebral caudal (C). Artéria cerebral média (M), artéria cerebral rostral (R).

téria cerebelar rostral, encuanto no outro, estão presentes apenas junto à origem da artéria cerebral caudal; apenass à esquerda, em 6 peças $(20,0 \% \pm 7,3$ Fig. 3), sendo yue, em 3 peças, a angulação situa-se junto à origem da artéria cerebral caudal esquerda, enquanto em outra localiza-se em correspondência à artéria cerebelar rostral esquerda c, nas duas últimas, no ponto de origem das artérias cerebral caludal esquerda e cerebelar rosiral esquerda; somente à direita, em 2 casos $(6,6 \% \pm 4,5$ Fig. 3), sendo que, em um deles está presente na origem da artéria cerebral caudal, encuanto no outro, encontra-se junto à origem da artéria cerchelar rostral. Em 2 preparações $(6,6 \% \pm 4,5$ Fig. 3) esta formação piriforme apresenta-se mais alongada que nas demais peças.

No grupo das 13 peças (Fig. 4) cuja figura é poligonal, 
ALCAN'TARA, M. A.: PRADA. I. L. S. Artérias da base do encéfalo de cães (Canis familiaris, linnacus, 1758). II. Formação e comportamento do circuito arterial do encéfalo. Bray, J. vet, Res, anim. Sci. Săo Paulo, y 33, n.2, 72-76, 1096.

destacamos 2 casos $(6,6 \% \pm 4,5$ Fig. 4$)$ em que o antímero direito da referida figura, representado pelo ramo caudal da artéria carótida interna, apresenta-se curvo. Verificamos ainda que, em caso único, o ramo terminal direito da artéria basilar, após dar origem à artéria cerebelar rostral apresenta concavidade voltada para fora do circuito arterial.

\section{DISCUSSĀO}

Quanto à particular disposição dos ramos terminais (rostral e caudal) das artérias carótidas internas esquerda e direita e dos ramos terminais da artéria basilar, constituindo figura que acabamos de descrever, tivemos grande dificuldade em encontrar designação apropriada relativa a essa formação. De fato, a figura que encontramos na maior parte dos casos $(56,6 \%)$ não corresponde nem a um polígono arterial, como referem Ellenberger; Baum5 (1894) e Baptista' (1922), nem a um hexágono, como citam Ellenberger; Baum6 (1977), nem tampouco ao círculo arterial ou arterioso, a que aludem De Vriese 4 (1905); Bradley ${ }^{2}$ (1948); Nickel et al." (1975) e Evans; Christensen ${ }^{7}$ (1979), ou círculo de Willis, como informam James; Hoerlein" (1960).

A formação que descrevemos constitui-se a partir da divisão da artéria carótida interna, de ambos os lados, em seus ramos terminais rostral e caudal; apresenta-se de modo invariável, rostralmente, em pequeno arco ou ferradura, de concavidade caudal. Entretanto, caudalmente mostra forma muito variada. Assim, em $56,6 \%$ das peças, constitui figura piriforme cuja base relaciona-se aos pedúnculos cerebrais. Esta figura, no seu todo, está longe de corresponder a um polígono, cujo conceito em geometria é o de uma figura que apresenta vários ângulos que somam $360^{\circ}$. Também não corresponde a um círculo, visto que este em geometria representa a região de um plano limitada por uma circunferência. Assim, preferimos adotar a expressão CIRCUITO ARTERIAL DO ENCÉFALO, uma vez que esta palavra "circuito", segundo


contorno, periferia, o que circunda, com significação, portanto, que atende à figura por nós encontrada na maioria dos casos (56,6\%), como também à própria figura que encontramos nos outros casos, $43,3 \%$, figuras essas que apresentam, em sua porção caudal, disposição respectivamente piriforme ou poligonal. Quanto à localização da formação que descrevemos a contornar o quiasma óptico e a hipófise, Ellenberger; Baums (1894); Baptista' (1922) e Evans; Christensen $^{7}$ (1979) referem-se a ela de maneira genérica. Segundo Baptista' (1922), este círculo dispõe-se em torno da sela túrcica, enquanto para Ellenberger; Baum $^{5}$ (1894) e Evans; Christensen $^{7}$ (1979) ela se encontra na superfície ventral do encéfalo, sendo que apenas Bradley² (1948) particulariza que se estende numa depressão em frrente à ponte.

No atinente aos vasos que colaboram para constituir a formação 
que estamos discutindo, fundamentalmente o apresentado por estes autores não difere do que descrevemos; o que verificamos é que utilizam nomenclatura diferente para os vasos implicados.

A disposição em ferradura, que representa a porção rostral do circuito arterial a que estamos aludindo, constituise, de cada lado, pelo ramo rosiral da artéria carótida interná, que se continua rostralmente com a artéria cerebral rostral correspondente. O que acabamos de descrever tem respaldo nas Figs. II-27 e II-28 de Evans; Christensen (1979) e na Fig. 5.5-1.3 de Nandal" (1986), nas quais observamos a mesma disposição em arco ou ferradura a que acabamos de nos relerir. Como, caudalmente, o que vemos nesta figura lembra um polígono (alongado), estranha-nos o fato de estes autores terem se referido a esta formação como círculo arterial; aliás, esses autores aludem à formação como um alongado anel arterial. Outra incongruência encontramos relativamente ao referido por Ellenberger;

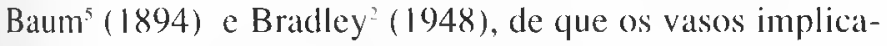
dos na circulação cerebral vão ter à base da cavidade cranial, formando um círculo que é representado pelo polígono de Willis.

A disposição em lïgura piriforme ou poligonal que descrevemos no alinente à porção catudal do circuito arterial é formada lateralmente pelos ramos caudais das artérias carótidas internas esquerda e direita até o local de origem da artéria cerebral caudal e, caudalmente, pelos ramos terminais da artéria basilar, referências estas que estão concordantes

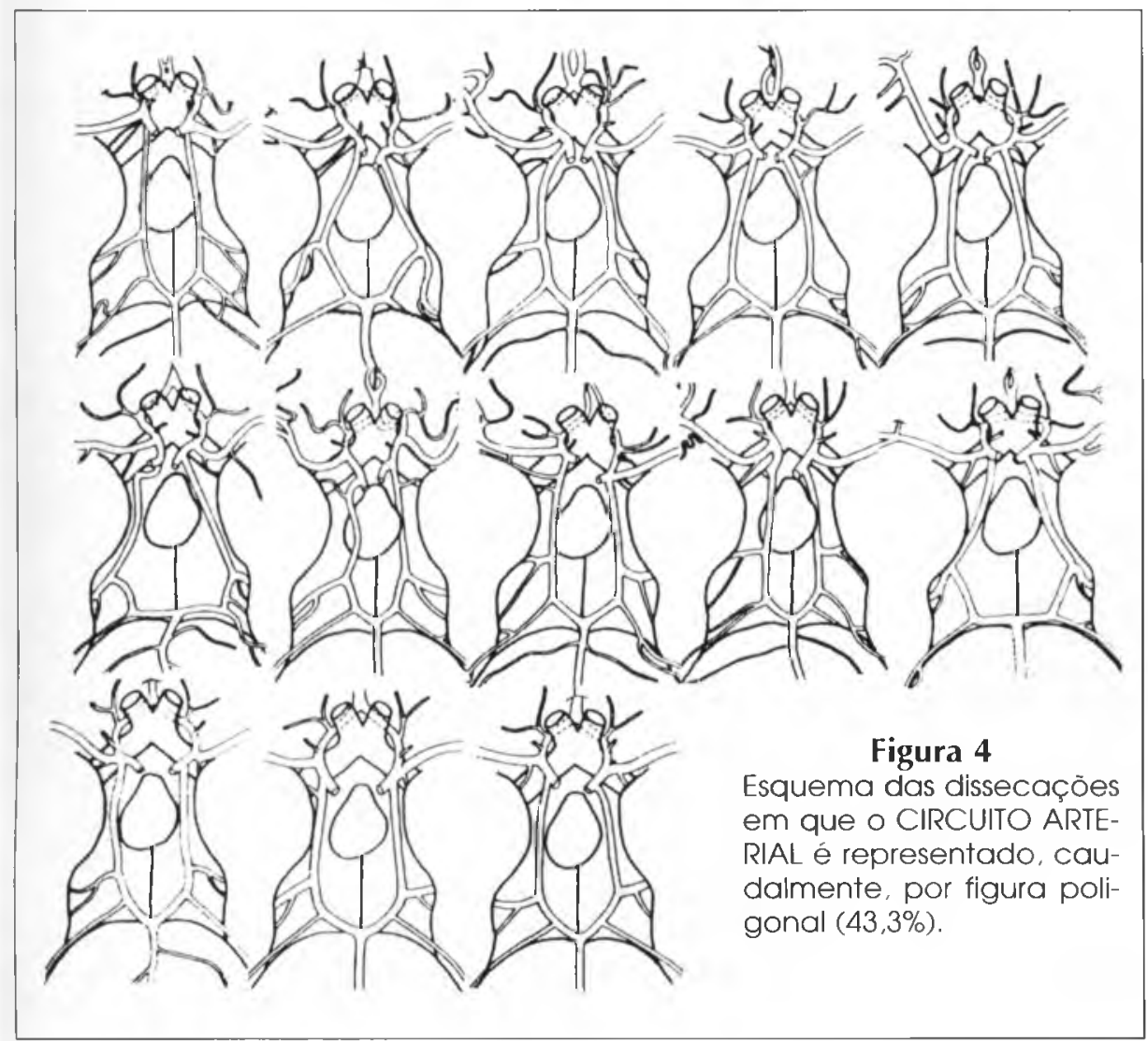

com as de Bradley² (1948), a esse respeito. Entretanto De La Torre et al.' ( 1959) e Evans; Christensen? (1979) referem-se a esses limites implicando o que chamam de artéria comunicante posterior. Discordamos desta designação que apenas tem sua razão de ser relativamente aos primatas, inclusive ao próprio homem (como pudemos verilïar nos estudos de Testut ${ }^{13}, 1911$ ), nos quais os dois sistemas (da artéria carótida interna e o da basilar), tendendo a separar-se, mantém ainda vínculo através desta artéria comunicante caudal. Entretanto, em absoluto, isso não se aplica aos cães, onde os dois sistemas constituem uma única unidade vascular, centralizada no circuito arterial.

Relativamente aos ramos terminais da artéria basilar, queremos lembrar o lato de que foi escolhido com muito cuidado o critério para identificá-los da maneira como o lïzemos; assim, eles foram considerados desde a bifurcação da artéria até a origem, em ambos os lados, da artéria cerebral caudal. Para tanto, huscamos ler a respeito da disposição das artérias da base do encéfalo, em outras espécies animais e no próprio homem, como também nas sequientes fases do desenvolvimento ontogenético: desta maneira, consultando De Vriese ${ }^{+}$ (1905), pudemos ler que nas fases iniciais do desenvolvimento ontogenético, os mamíleros mostram o tipo arterial dos vertebrados inferiores, nos quais as artérias carólidas internas representam as únicas artérias cerebrais. Os estágios embrionários mais avançàdos modificam-se no sentido de que as artérias vertebrais se unem secundariamente às artérias carótidas e a evolução caminha em direção ao tipo mais recente, no yual as artérias vertebrais avançam relativamente ao território carotídeo cerebral. Entendemos que esse avançar do sistema vértebro-basilar em relação ao sistema carotídeo implica basicamente o território da artéria cerebral caudal que, inicialmente era da responsabilidade da artéria carótida interna passando, depois, para o domínio da artéria basilar. Segundo nossa interpretação, a artéria cercbral caudal, nos cães estudados, não se evidencia ainda como continuação direta do ramo terminal da artéria basilar, representando, portanto, o limite entre o ramo caudal da artéria carótida interna e o ramo terminal da artéria basilar.

Face ao exposto, propomos à $\mathrm{Co}$ missão Internacional de Nomenclatura Anatômica cue a formação vascular ora enfocada, em cães, passe a ter a designação de "Circuito Arterial do Encéfalo".

\section{CONCIUSÕES}

1) A particular disposição dos ramos 


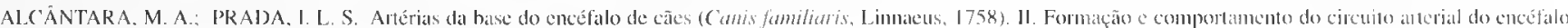
Braz. .J. vet. Res. anim. Sci. São Paulo, v..33, 11.2, p.72-76, 1996.

terminais das artérias carótidas internas esquerda e direila e dos ramos terminais da artéria basilar determina formação que contorna o quiasma óptico e a hipófise. Essa formação, a partir da divisão da artéria carótida interna, de ambos os lados, em seus ramos terminais (rostral e caludal), apresenta-se de modo invariável rostralmente, em pequeno areo ou lerradura de concavidade caudal e, catudalmente de forma variada, constituindo ligura piri- forme $(56,6 \%)$ ou poligonal $(43,3 \%)$.

2) Essa fomação, no seu todo, não corresponde nem a um polígono nem a um círculo, tendo sido, portanto, até o momen1o, impropriamente designada com esses termos. Assim, tornase imperativo que a Comissão Intemacional de Nomenclatura Anatômica reàvalic a denominação dessa forma Vascular, em cães. Nossa proposta, face ao exposto, é a de que, para lanto, se adote a designação de "Circuito Arterial do Encélalo".

\section{SUMMARY}

For this research, 30 pieces of crossbed dogs were dissected, in order to improve our knowledge on the behaviour of the arteries in the basis of the encephalon.

The preparations showed that the peculiar disposal of the terminal branches of the internal carotid, and the terminal branches of the basilar artery determine, starting from the splitting of the internal carotid artery -- in both sides -into rostral and caudal terminal branches, this formation invariably displays the shape of a small arc, or that of a horseshoe with caudal concavity. Caudally, it forms, in variable ways, pyriform $(56,6 \%)$ or poligonal $(43,3 \%)$ pictures. a) rostrally represented by left and right rostral cerebral arteries; b) laterally, by the rostral and caudal branches of left and right internal carotid arteries; c) caudolaterally, by left and right terminal branches of the basilar artery.

UNITERMS: Anatomy; Dogs; Brain; Arteries.

\section{REFERÊNCIAS BIBLIOGRÁFICAS}

I-BAI'TISTA. B.V. Fstudo comparado dá circulação cerebral nos mamiferos domésticos e no homem - Razáo de ser da rede admirável. Rio de Janciro. 1922. Tese (Doutorado) - Faculdade de Medicina do Rio de Janciro.

2-BRAILEY, O.C. Topographical anatomy of the dog. 5 ed. London. lidinghurgh TweeddaleCourt. 1948. p. 264-7.

3-1)E I A TORRE: E.; NITSKY. M.G.: MESCIIAN. I. Intrateranial and extracrintal circulation in the dog: anatomic and angiograliestudies. American Journal Anatomy, v. I(05, p. 343-81, 1959.

4 DE VRIESE. B. Sur la signification morlologiçue des artères cérúbrales. Archives de Biologie, v.21, p.357-457, 1905.

5-ELLLENBERGER, W.: BAUM, II. Anatomie descritive et topographique du chien. Paris, C. Reinwald. Librairies Editeurs, 1894 p. $378-82$.

6-IILLENBERG IER, W. BALIM. I I Iandbuch der vergleicheden Analomie der Hausliere. Berlin. Julius Springer, 1977. p.672-6.

7-liVANS. II.E.; CHIRISTENSEN. (i.C. Miller's Anatomy of the dog. Philadcllia. W.B. Saunders, 1979, p.656-87.

8-IERREIRA, A.B.JI. Novo dicionário da língua portuguesa. 2. ed. Rio de Janciro, Nova Ironteira, 1986, p.4I0, 1356.

4-JAMES, W.V.: IIOERI.EIN. B.F. Cerebral angiography in the dog Velerinary Medicine, p.45-56, I960).

10 NANDA. B.S. Suprmento sanguiineo paran o cérebro. In: (iE'T'TY, R.; SISSON, S.: (GROSSMAN, J.D. Anatomia dos animais domésticos. 6. ed. Rio de Janeiro, Cinanabarat. Koogant. 1986. v.2. p.1513-8.

I1-NICKEI, R.: SCIIIIMER, A.; SEIFERLE, E. Nervensystem Sinnessorgane Indokrine Drüsen. Berlim. Paul Parey, 1975. v.4. P. 174-81

12-NOMINA ANATOMIC A VETERINARIA. 3.ed. New York. International Committe on Veterinary Gross Anatomical momenclature. 1983.

13-TESTUT, L. Traité d'anatonie humaine. G.ed. Paris, Octave Dorn, 1911. v.2, p.917.20. 